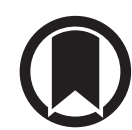

CrossMark

\section{Abnormal carbon monoxide diffusion capacity in COVID-19 patients at time of hospital discharge}

\author{
To the Editor:
}

I have read with great interest the article by Mo et al. [1] entitled "Abnormal pulmonary function in COVID-19 patients at time of hospital discharge" recently published in the European Respiratory Journal. In this study, the authors describe pulmonary function tests in patients who suffered from coronavirus disease 2019 (COVID-19), which were performed on discharge from hospital. Patients were classified into three groups of severity. In the patients who suffered more severely, measured carbon monoxide diffusion capacity $\left(D_{\mathrm{LCO}}\right)$ was lower. However, when looking at $D_{\mathrm{LCO}} / V_{\mathrm{A}}$, which represents the transfer factor of carbon monoxide, the picture is different. Patients who had severe pneumonia had an average $D_{\mathrm{LCO}} / V_{\mathrm{A}}$ of $82 \%$ of predicted while patients in groups classified as having mild disease or pneumonia had average values above $90 \%$. Notably, all these averages have relatively high standard deviation values (e.g. $13.9 \%$ in severe pneumonia), meaning that some patients in the post-severe pneumonia group had a $D_{\mathrm{LCO}} / V_{\mathrm{A}}>90 \%$ of predicted.

The authors present timely and important data as questions are now being raised concerning the long-term effects of COVID-19 [2]. However, I disagree with the authors concerning their interpretation of both the diffusion data and also what their findings mean clinically. In presenting their results the authors use the phrase " $D_{\mathrm{LCO}}$ corrected for alveolar volume $\left(D_{\mathrm{LCO}} / V_{\mathrm{A}}\right)$ " which is not a correct expression and the notion of " $D_{\text {LCO }}$ corrected for alveolar volume" is not acceptable these days [3]. The authors explain further that " $D_{\mathrm{LCO}} / V_{\mathrm{A}}$ was still within the normal range, which might indicate that $D_{\mathrm{LCO}}$ decrease was more than the $D_{\mathrm{LCO}} / V_{\mathrm{A}}$ in recovered subjects", which is actually restating a finding without drawing a conclusion.

It is important to give emphasis here that by using carbon monoxide, $D_{\mathrm{LCO}}$ represents the gas diffusion capacity across the alveolar-capillary barrier and is in fact a mathematical product of the rate constant representing alveolar carbon monoxide uptake efficiency $\left(D_{\mathrm{LCO}} / V_{\mathrm{A}}\right)$ multiplied by alveolar volume $\left(V_{\mathrm{A}}\right)$ [3]. Thus, when $D_{\mathrm{LCO}} / V_{\mathrm{A}}$ is normal or near normal, $D_{\mathrm{LCO}}$ may still be low because of reduced alveolar volume, which means that complete acini with alveoli and blood vessels surrounding them are affected [3, 4]. In the remaining functionally available alveolar volume, the actual carbon monoxide uptake is normal as the diffusion through the alveolar-capillary barrier is not impaired and the blood volume within the pulmonary capillary bed is not reduced. Interstitial abnormalities or pulmonary vascular abnormalities are associated with reduced $D_{\mathrm{LCO}} / V_{\mathrm{A}}$ which accompanies reduced $D_{\mathrm{LCO}}[4]$.

In the discussion of their findings, the authors cite previous studies describing follow-up of severe acute respiratory syndrome (SARS) patients having impaired $D_{\mathrm{LCO}}$ as the most common abnormality, with affected proportion of patients ranging from $15.5 \%$ to $43.6 \%$. It would be important to describe how impaired $D_{\mathrm{LCO}}$ was defined in those studies, citing values as $\%$ of predicted and comparing that with the findings of the present study.

The conclusion which can be obtained from this study is that low $D_{\text {LCO }}$ is caused mainly by reduced alveolar volume, and not residual interstitial abnormalities or pulmonary vascular abnormalities caused by COVID-19. The latter is important, since the proposed pathogenic mechanism describes an initial abnormality induced by COVID-19 similar to the effect of SARS and consists of a microvascular injury with some interstitial thickening with clear lungs on imaging and physical examination accompanied by profound hypoxaemia $[5,6]$, followed by development of alveolar abnormalities with consequent gradual

@ERSpublications

On recovery from COVID-19 it is important to draw attention to the CO diffusion test and the actual meaning of the findings when considering the values of $D_{\mathrm{LCO}}$ and $D_{\mathrm{LCO}} / V_{\mathrm{A}}$ put together https://bit.ly/36k2O2Q

Cite this article as: Nusair S. Abnormal carbon monoxide diffusion capacity in COVID-19 patients at time of hospital discharge. Eur Respir J 2020; 56: 2001832 [https://doi.org/10.1183/13993003.01832-2020]. 
loss of the alveolar spaces [6]. The filling of the alveoli with exudate at a later stage causes the loss of lung volume and reduced pulmonary compliance [7]. The finding of decreased alveolar volume on discharge may be explained by transient changes in mechanical properties of the chest wall and respiratory muscles after critical illness and may well address the concerns about a possible long lasting pulmonary parenchymal dysfunction post-COVID-19 [2].

In conclusion, the authors presented very useful data, but it is important to draw attention to the up-to-date manner in which we look at the carbon monoxide diffusion test and the actual meaning of the findings when considering the results of $D_{\mathrm{LCO}}$ and $D_{\mathrm{LCO}} / V_{\mathrm{A}}$ together.

Samir Nusair $\circledast^{1,2}$

${ }^{1}$ Rokach Center for the Prevention of Lung Diseases, Clalit Health Services, Jerusalem Region, Affiliated to The Faculty of Medicine, Hebrew University and Hadassah, Jerusalem, Israel. ${ }^{2}$ Institute of Pulmonary Medicine, Hadassah-Hebrew University Medical Center, Jerusalem, Israel.

Correspondence: Samir Nusair, Institute of Pulmonary Medicine, Hadassah-Hebrew University Medical Center, PO Box 12000, Jerusalem 91120, Israel. E-mail: samjack@actcom.co.il

Received: 17 May 2020 | Accepted: 18 May 2020

Conflict of interest: S. Nusair has nothing to disclose.

\section{References}

1 Mo X, Jian W, Su Z, et al. Abnormal pulmonary function in COVID-19 patients at time of hospital discharge. Eur Respir J 2020; 55: 2001217.

2 Spagnolo P, Balestro E, Aliberti S, et al. Pulmonary fibrosis secondary to COVID-19: a call to arms? Lancet Respir 2020; in press [https://doi.org/10.1016/S2213-2600(20)30222-8].

3 Hughes JMB, Pride NB. Examination of the carbon monoxide diffusing capacity (DLCO) in relation to its KCO and VA components. Am J Respir Crit Care Med 2012; 186: 132-139.

4 Ayers LN, Ginsberg ML, Fein J, et al. Diffusing capacity, specific diffusing capacity and interpretation of diffusion defects. West J Med 1975; 123: 255-264.

5 McGonagle D, O’Donnell JS, Sharif K, et al. Immune mechanisms of pulmonary intravascular coagulopathy in COVID-19 pneumonia. Lancet Rheumatol 2020; in press [https://doi.org/10.1016/S2665-9913(20)30121-1].

6 Tian S, Hu W, Niu L, et al. Pulmonary pathology of early-phase 2019 novel coronavirus (COVID-19) pneumonia in two patients with lung cancer. J Thorac Oncol 2020; 15: 700-704.

7 Zhou F, Yu T, Du R, et al. Clinical course and risk factors for mortality of adult inpatients with COVID-19 in Wuhan, China: a retrospective cohort study. Lancet 2020; 395: 1054-1062.

\section{Impaired pulmonary function in discharged patients with COVID-19: more work ahead}

From the authors:

We are grateful for the opportunity to respond to the letter by S. Nusair, whom we thank for his interest and thoughtful remarks about our study on the abnormal pulmonary function in coronavirus disease 2019 (COVID-19) patients [1].

@ERSpublications

Impairment of diffusion capacity is the most common abnormality in discharged patients with COVID-19. Both decreased alveolar volume and $K_{\mathrm{CO}}$ contribute to the pathogenesis of impaired diffusion capacity. More follow-up work is needed. https://bit.ly/2YL2eaK

Cite this article as: Chen $\mathrm{R}, \mathrm{Gao} \mathrm{Y}$, Chen $\mathrm{M}$, et al. Impaired pulmonary function in discharged patients with COVID-19: more work ahead. Eur Respir J 2020; 56: 2002194 [https://doi.org/10.1183/ 13993003.02194-2020]. 
Diffusing capacity of the lung for carbon monoxide $\left(D_{\mathrm{LCO}}\right)$ reflects the capacity of carbon monoxide transfer from the environment to the pulmonary capillary blood, which is the most clinically useful routine methodology to evaluate the function of the lung to exchange gas. $K_{\mathrm{CO}}$ is the rate constant for carbon monoxide uptake from alveolar gas, which is influenced mostly by the thickness and area of the alveolar capillary membrane, the volume of blood in capillaries supplying ventilated alveoli, and the concentration and properties of haemoglobin in the alveolar capillary blood. $D_{\mathrm{LCO}}$ is mainly determined by $K_{\mathrm{CO}}$ and the alveolar volume $\left(V_{\mathrm{A}}\right)$. Mathematically, $K_{\mathrm{CO}}$ can be calculated as $D_{\mathrm{LCO}} / V_{\mathrm{A}}$ under body temperature, ambient pressure, saturated with water vapour conditions [2].

We agree with $\mathrm{S}$. Nusair that it should be noted that $D_{\mathrm{LCO}} / V_{\mathrm{A}}$ is not a simple ratio and in fact the relationship between lung volume and carbon monoxide uptake is linear and certainly less than 1:1 [3]. Recent studies have tended to use $K_{\mathrm{CO}}$ instead of $D_{\mathrm{LCO}} / V_{\mathrm{A}}$, as it may be inferred from the use of the term $D_{\mathrm{LCO}} / V_{\mathrm{A}}$ that $D_{\mathrm{LCO}}$ can be normalised for $V_{\mathrm{A}}$. The 2017 European Respiratory Society/American Thoracic Society standards for single-breath carbon monoxide uptake in the lung recommends that the term of $K_{\mathrm{CO}}$ is preferred to $D_{\mathrm{LCO}} / V_{\mathrm{A}}$ [2]. However, in practice, many pulmonary function test reports from different manufacturers still present with $D_{\mathrm{LCO}} / V_{\mathrm{A}}$ and not $K_{\mathrm{CO}}$. Besides, in order for an easier and direct comparison with some previous studies on pulmonary function in patients with severe acute respiratory syndrome (SARS) $[4,5], D_{\mathrm{LCO}} / V_{\mathrm{A}}$ was kept in our report. It is important to understand the interpretation of $K_{\mathrm{CO}}$ and $D_{\mathrm{LCO}} / V_{\mathrm{A}}$, but the term of $K_{\mathrm{CO}}$ is recommended for more popular use in future.

Decreased $K_{\mathrm{CO}}$ occurs in alveolar-capillary damage, microvascular pathology or anaemia. Causes of low $V_{\mathrm{A}}$ include reduced alveolar expansion, alveolar damage or loss, or inspired gases maldistributed with airflow obstruction. When $K_{\mathrm{CO}}$ turns normal, in the presence of a low $D_{\mathrm{LCO}}$ it is associated with low $V_{\mathrm{A}}$. Because only the functional alveolar units had been sampled, a biased picture toward more preserved areas of the lungs is thereby provided [3]. Crucially, the same $D_{\mathrm{LCO}}$ may occur with various combinations of $K_{\mathrm{CO}}$ and $V_{\mathrm{A}}$, each suggesting different pathologies. It is difficult to interpret which is the predominant role because both decreased alveolar volume and $K_{\mathrm{CO}}$ contribute to the pathogenesis of impaired diffusion capacity.

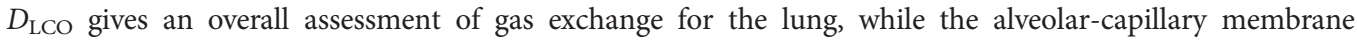
diffusing capacity for carbon monoxide $\left(D_{\mathrm{MCO}}\right)$ only dependents on molecular diffusion of the membranes. However, $D_{\mathrm{MCO}}$ can be calculated using different equations but is not yet standardised, and as such is not a commonly used gas diffusing parameter [6].

In our study, 51 patients with COVID-19 had impaired $D_{\mathrm{LCO}}$, including 29 patients who showed both impaired $D_{\mathrm{LCO}}$ and $K_{\mathrm{CO}}$. The number of patients with both impaired $D_{\mathrm{LCO}}$ and $K_{\mathrm{CO}}(29 / 51)$ was $24 \%$ larger than the number of patients with impaired $K_{\mathrm{CO}}$ alone (22/51). Moreover, the percentage of patients with decreased total lung capacity was less than the percentage of patients with decreased $K_{\mathrm{CO}}$. Furthermore, vascular injury and thrombosis have been demonstrated to be important contributing factors in the pathogenesis of COVID-19. SARS coronavirus 2 infects the endothelial cells via angiotensin-converting enzyme 2 . The recruitment of immune cells to the endothelial cells facilitates the induction of endotheliitis in several organs, which contributes to systemic impaired microcirculatory function [7]. Acute pulmonary embolism has been reported during the clinical course [8]. Post mortemstudies have reported vascular injury and thrombi in multiple organs [7,9]. ACKermann et al. [10] compared the lung pathology of COVID-19 with H1N1. The result showed that patient with COVID-19 presented distinctive vascular features, consisting of severe endothelial injury associated with the disruption of intercellular junctions, cell swelling and a loss of contact with the basal membrane. From the above, it is probable that the effect of membrane lesion plays an important role on the impaired diffusion capacity in patients with COVID-19.

Our study showed that, similar to $D_{\mathrm{LCO}}, D_{\mathrm{LCO}} / V_{\mathrm{A}}$ (representing the transfer factor of carbon monoxide) also showed an association with the disease severity: as the patients progressed to a more severe condition, the mean value of $D_{\mathrm{LCO}} / V_{\mathrm{A}}$ compared to predicted values was lower. Since the standard deviation values suggest that individual differences have effects on lung function, we also provided the distribution of the $D_{\mathrm{LCO}} / V_{\mathrm{A}}$ of predicted in patients with different severities. $42 \%$ of the subjects with severe pneumonia had a $D_{\mathrm{LCO}} / V_{\mathrm{A}}$ of less than $80 \%$ of predicted, compared to only $27 \%$ in the patients with pneumonia and $13 \%$ in patients with mild illness, respectively.

In previous follow-up studies of SARS, impaired $D_{\mathrm{LCO}}$ was defined as when $D_{\mathrm{LCO}}$ was $<80 \%$ of the predicted value, irrespective of $K_{\mathrm{CO}}$ or the alveolar volume. In follow-up studies of rehabilitating SARS patients ranging from 0.5 to 2 years, the impaired $D_{\mathrm{LCO}}$ was the most common abnormality, accounting for $15.5 \%$ to $43.6 \%$ [11-13]. The mean $D_{\text {LCO }}$ ranged from $77.7 \%$ to $93.8 \%$ pred in the studies above, with the longest follow up of 2 years. Our study showed a mean $D_{\mathrm{LCO}}$ of $78.2 \%$ pred at time of discharge but follow-up data is not available yet. It is unclear how the impaired diffusion capacity changes in survivors of COVID-19, as well as how the changes may be reflected on chest computed tomography (CT). In one study, where surviving SARS 
patients were seen 6 months after admission, 43(75.4\%) of patients showed radiological abnormalities, including $62.8 \%$ who had abnormal $D_{\mathrm{LCO}}$ [11]. Thus, the lung function impairment findings are consistent with the CT images of SARS survivors showing persistent ground glass opacity, reticular opacities and traction bronchiectasis suggesting the fibrosis [14]. Likewise, in discharged patients with COVID-19, WANG et al. [15] reported that $94 \%(66 / 70)$ of patients who were discharged from hospital at the end of the study still had mild to substantial residual lung abnormalities on their last CT scans.

Our study was a pilot study which first described the impaired pulmonary function in patients with COVID-19 at time of discharge. Impairment of diffusion capacity is the most common abnormality followed by restrictive ventilatory defects. However, more follow-up work is needed to assess the longitudinal variation of these deficits.

Ruchong Chen ${ }^{1}$, Yi Gao ${ }^{1}$, Mu Chen ${ }^{2}$, Wenhua Jian ${ }^{1}$, Chunliang Lei ${ }^{2}$, Jinping Zheng ${ }^{1}$ and Shiyue $\mathrm{Li}^{1}$

${ }^{1}$ State Key Laboratory of Respiratory Disease, National Clinical Research Center for Respiratory Disease, Guangzhou Institute of Respiratory Health, First Affiliated Hospital of Guangzhou Medical University, Guangzhou, China. ${ }^{2}$ Dept of Respiratory Medicine, Guangzhou Eighth People’s Hospital, Guangzhou, China.

Correspondence: Shiyue Li, State Key Laboratory of Respiratory Disease, National Clinical Research Center for Respiratory Disease, Guangzhou Institute of Respiratory Health, First Affiliated Hospital of Guangzhou Medical University, Guangzhou, China. E-mail: lishiyue@188.com

Received: 6 June 2020 | Accepted: 11 June 2020

Conflict of interest: R. Chen has nothing to disclose. Y. Gao has nothing to disclose. M. Chen has nothing to disclose. W. Jian has nothing to disclose. C. Lei has nothing to disclose. J. Zheng has nothing to disclose. S. Li has nothing to disclose.

\section{Reference}

1 Mo X, Jian W, Su Z, et al. Abnormal pulmonary function in COVID-19 patients at time of hospital discharge. Eur Respir J 2020; 55: 2001217.

2 Graham BL, Brusasco V, Burgos F, et al. 2017 ERS/ATS standards for single-breath carbon monoxide uptake in the lung. Eur Respir J 2017; 49: 1600016.

3 Neder JA, Berton DC, Muller PT, et al. Incorporating lung diffusing capacity for carbon monoxide in clinical decision making in chest medicine. Clin Chest Med 2019; 40: 285-305.

4 Ong K-C, Ng AW-K, Lee LS-U, et al. 1-year pulmonary function and health status in survivors of severe acute respiratory syndrome. Chest 2005; 128: 1393-1400.

5 Xie L, Liu Y, Xiao Y, et al. Follow-up study on pulmonary function and lung radiographic changes in rehabilitating severe acute respiratory syndrome patients after discharge. Chest 2005; 127: 2119-2124.

6 Zavorsky GS, Hsia CCW, Hughes JMB, et al. Standardisation and application of the single-breath determination of nitric oxide uptake in the lung. Eur Respir J 2017; 49: 1600962.

7 Varga Z, Flammer AJ, Steiger P, et al. Endothelial cell infection and endotheliitis in COVID-19. Lancet 2020; 395: 1417-1418.

8 Danzi GB, Loffi M, Galeazzi G, et al. Acute pulmonary embolism and COVID-19 pneumonia: a random association? Eur Heart J 2020; 41: 1858-1858.

9 Reichard RR, Kashani KB, Boire NA, et al. Neuropathology of COVID-19: a spectrum of vascular and acute disseminated encephalomyelitis (ADEM)-like pathology. Acta Neuropathol 2020; 5: 336-336.

10 Ackermann M, Verleden SE, Kuehnel M, et al. Pulmonary vascular endothelialitis, thrombosis, and angiogenesis in Covid-19. N Engl J Med 2020; 383: 120-128

11 Ng CK, Chan JWM, Kwan TL, et al. Six month radiological and physiological outcomes in severe acute respiratory syndrome (SARS) survivors. Thorax 2004; 59: 889-891.

12 Hui DS, Wong KT, Ko FW, et al. The 1-year impact of severe acute respiratory syndrome on pulmonary function, exercise capacity, and quality of life in a cohort of survivors. Chest 2005; 128: 2247-2261.

13 Ngai JC, Ko FW, Ng SS, et al. The long-term impact of severe acute respiratory syndrome on pulmonary function, exercise capacity and health status. Respirology 2010; 15: 543-550.

14 Chang Y-C, Yu C-J, Chang S-C, et al. Pulmonary sequelae in convalescent patients after severe acute respiratory syndrome: evaluation with thin-section CT. Radiology 2005; 236: 1067-1075.

15 Wang Y, Dong C, Hu Y, et al. Temporal changes of CT findings in 90 patients with COVID-19 pneumonia: a longitudinal study. Radiology 2020; in press [https://doi.org/10.1148/radiol.2020200843]. 